\title{
Microscopic calculation of the inclusive electron scattering structure function in ${ }^{16} \mathrm{O}$
}

\author{
Bogdan Mihaila* \\ Department of Physics, University of New Hampshire, Durham, NH 03824 \\ Physics Division, Oak Ridge National Laboratory, P.O. Box 2008, Oak Ridge, TN 37831 \\ Jochen H. Heisenbergit \\ Department of Physics, University of New Hampshire, Durham, NH 03824
}

(July 1, 2021)

\begin{abstract}
We calculate the charge form factor and the longitudinal structure function for ${ }^{16} \mathrm{O}$ and compare with the available experimental data, up to a momentum transfer of $4 \mathrm{fm}^{-1}$. The ground state correlations are generated using the coupled cluster $[\exp (\mathbf{S})]$ method, together with the realistic v18 NN interaction and the Urbana IX three-nucleon interaction. Centerof-mass corrections are dealt with by adding a center-of-mass Hamiltonian to the usual internal Hamiltonian, and by means of a many-body expansion for the computation of the observables measured in the center-of-mass system.
\end{abstract}

PACS numbers: 24.10.Cn,25.30.-c,21.60.Gx,27.20.+n

One of the fundamental problems in nuclear physics is related to developing a complete understanding of how nuclear structure arises as a result of the underlying interaction between nucleons. This in turn should help us develop a complete understanding of the electromagnetic structure of the nucleus, as revealed by the wealth of high-quality data that electron scattering experiments have provided for the past 30 years. The interplay of nuclear correlations, meson-exchange current or charge densities, relativistic effects in nuclei, the importance of three- or more many-body interactions in relation with the dominant two-body interaction in nuclei awaits to being assessed in greater detail. Unfortunately, solutions of the many-body Schrödinger equation with realistic interactions have been proven very difficult to obtain. Only in recent years has progress been made and first results of microscopic calculations relating to ground-state and low-excited states for nuclei with $A \leq 7$ been reported [1]. These calculations have been obtained using the Green's function Monte Carlo method, but this approach, just like the Fadeev [2] or the Correlated Hyperspherical Harmonics [3] methods successfully used for the $A=3,4$ body system, suffers limitations in the number of nucleons they can treat. To date, only the Variational Monte Carlo method 4 has enjoyed success in solving the manybody problem for medium nuclei, but those results still show a room for improvement.

We are using the $\exp (\mathbf{S})$ coupled-cluster expansion to calculate the ground state of ${ }^{16} \mathrm{O}$. Our approach is very similar to the standard approach, first developed by the Bochum group [5], and has been outlined recently in [8].

*electronic mail:Bogdan.Mihaila@unh.edu

†electronic mail:Jochen.Heisenberg@unh.edu
The idea behind the coupled-cluster expansion formalism relies on the ability of expanding the model nuclear wave function in the many-body Hilbert space in terms of two Abelian subalgebras of multiconfigurational creation and their Hermitian-adjoint destruction operators. The expansion coefficients carry then the interpretation of nuclear correlations. The fact that we make no artificial separation between "short-range" and "long-range" correlations is one particular strength of this many-body method.

The derivation of the explicit equations is quite tedious, but requires only standard techniques. For a closed-shell nuclear system, the total Hamiltonian is given as

$$
\mathbf{H}=\sum_{i} T_{i}+\sum_{i<j} V_{i j}+\sum_{i<j<k} V_{i j k}^{t n i} .
$$

The Hamiltonian includes a nonrelativistic one-body kinetic energy, a two-nucleon potential, and a supplemental three-nucleon potential. We have chosen the Argonne $v 18$ potential [6] as the most realistic nucleon-nucleon interaction available today. The Argonne $v 18$ model provides an accurate fit for both $p p$ and $n n$ scattering data up to $350 \mathrm{MeV}$ with a $\chi^{2} /$ datum near one. The introduction of charge-independence breaking in the strong force is the key element of obtaining this high performance. However, the two-body part of this interaction results in over-binding and too large a saturation density in nuclear matter. Therefore, the $N N$ potential is supplemented by a three-nucleon interaction (part of the Urbana family (7/), which includes a long-range two-pion exchange and a short-range phenomenological component. The Urbana-IX potential is adjusted to reproduce the binding energy of ${ }^{3} \mathrm{H}$ and give reasonable saturation density in nuclear matter when used with Argonne $v 18$ [1]. 
We are searching for the correlated ground state of the Hamiltonian $H$, which we denote by $|\tilde{0}\rangle$. The ansatz for the many-body wave function $|\tilde{0}\rangle$ is defined as the result of the cluster correlation operator, $\mathrm{S}^{\dagger}$, acting on the reference state of the many-body system, the uncorrelated ground state $|0\rangle$ :

$$
|\tilde{0}\rangle=e^{\mathbf{S}^{\dagger}}|0\rangle
$$

For a number-conserving Fermi system, the standard choice for $|0\rangle$ is the single-particle shell-model (Slater determinant) state formed from an antisymmetrized product of single-particle wave functions. The cluster correlation operator is defined in terms of its $p h$ creation operators expansion $\left(\mathbf{O}_{0}^{\dagger}=\mathbf{1}, \mathbf{O}_{1}^{\dagger}=\mathbf{a}_{p_{1}}^{\dagger} \mathbf{a}_{h_{1}}\right.$, $\left.\mathbf{O}_{2}^{\dagger}=\mathbf{a}_{p_{1}}^{\dagger} \mathbf{a}_{p_{2}}^{\dagger} \mathbf{a}_{h_{2}} \mathbf{a}_{h_{1}}\right)$ as

$$
\mathbf{S}^{\dagger}=\sum_{n=0}^{\infty} \frac{1}{n !} S_{n} \mathbf{O}_{n}^{\dagger}
$$

The problem of solving for the many-body wave function $|\tilde{0}\rangle$ and the ground-state energy, $E$, is now reduced to the problem of solving for the amplitudes $S_{n}$. This implies solving a set of non-linear equations, which may be obtained using a variational principle. We construct a variation $\delta|\tilde{0}\rangle$ orthogonal to the correlated ground state as

$$
\delta|\tilde{0}\rangle=e^{-\mathbf{S}} \mathbf{O}_{n}^{\dagger}|0\rangle
$$

and require that the Hamiltonian between the ground state and such a variation vanishes. As a result, we obtain an equation for the ground-state energy eigenvalue $E$ in terms of the cluster correlation coefficients, $\left\{S_{n}\right\}$, and a set of formally exact coupled nonlinear equations for these coefficients:

$$
\begin{aligned}
E & =\left\langle\tilde{0}\left|e^{\mathbf{S}} \mathbf{H} e^{-\mathbf{S}}\right| \tilde{0}\right\rangle, \\
0 & =\left\langle 0\left|e^{\mathbf{S}} \mathbf{H} e^{-\mathbf{S}} \mathbf{O}_{n}^{\dagger}\right| 0\right\rangle .
\end{aligned}
$$

Then, the computation breaks down into two steps [8]: In the first step, the G-matrix interaction is calculated inside the nucleus including all the corrections. This results in amplitudes for the $2 p 2 h$ correlations, which are implicitly corrected for the presence of $3 p 3 h$ and $4 p 4 h$ correlations. In the second step the mean field is calculated from these correlations and the single-particle Hamiltonian is solved to give mean-field eigenfunctions and single-particle energies. These two steps are iterated until a stable solution is obtained. Calculations are carried out entirely in configuration space where a $50 \hbar \omega$ space is used. The general approach, when the Hamiltonian includes only up to two-body operators, has been presented in 8.9.9. The results we report here have been obtained by taking into account the three-nucleon interaction via a density-dependent approach. The details of this approach will be presented elsewhere 10 .
Once the correlated ground state $|\tilde{0}\rangle$ is obtained, we can calculate the expectation value of any arbitrary operator $A$ as

$$
\bar{a}=\left\langle 0\left|e^{\mathbf{S}} A e^{-\mathbf{S}} \tilde{\mathbf{S}}^{\dagger}\right| 0\right\rangle,
$$

where $\tilde{\mathbf{S}}^{\dagger}$ is also defined by its decomposition in terms of ph-creation operators

$$
\tilde{\mathbf{S}}^{\dagger}=\sum_{n} \frac{1}{n !} \tilde{S}_{n} \mathbf{O}_{n}^{\dagger} .
$$

The amplitudes $\tilde{S}_{n}$ are obtained in terms of the $S_{n}$ amplitudes in an iterative fashion.

Note that the correlated ground state $|\tilde{0}\rangle$ is not translationally invariant since it depends on the $3 \mathrm{~A}$ coordinates of the nucleons in the laboratory frame. Therefore, in practice one has to take special care of correcting for the effects of the center-of-mass motion. This is done in several steps: First, the Hamiltonian (11) is replaced by the internal Hamiltonian

$$
H_{\text {int }}=H-T_{C M},
$$

which is now entirely written in the center-of-mass frame by removing the center-of-mass kinetic energy, $T_{C M}=$ $P_{C M}^{2} /(2 m A)$, with $m$ the nucleon mass. Both the twoand three-nucleon interactions are given in terms of the relative distances between nucleons, so in this respect no corrections are needed. Secondly, a many-body expansion has been devised [11] in order to carry out the necessary corrections required by the calculation of observables, which are measured experimentally in the centerof-mass frame. This procedure is based on the assumption that we can neglect the correlations between the center-of-mass and relative coordinates degrees of freedom, and a factorization of the correlated ground state $|\tilde{0}\rangle$ into components which depend only on the center-ofmass and the relative coordinates, respectively, is possible. We also assume that, indeed, the correlated ground state $|\tilde{0}\rangle$ provides a good description of the internal structure of the nucleus. Finally, in order to ensure such a separation, a supplemental center-of-mass Hamiltonian is added

$$
H_{C M}=\beta_{C M}\left[T_{C M}+\frac{1}{2}(m A) \Omega^{2} R_{C M}^{2}\right],
$$

which has the role of constraining the center-of-mass component of the ground-state wave function [12]. We choose the values of the parameters $\beta_{C M}$ and $\Omega^{2}$ such that they correspond to a value domain for which the binding energy

$$
E=\left\langle H_{i n t}^{\prime}=H_{i n t}+H_{C M}\right\rangle-\left\langle H_{C M}\right\rangle
$$

is relatively insensitive to the choice of the $\beta_{C M}$ and $\Omega^{2}$ values 10 . When leaving out the center-of-mass Hamiltonian, the calculated binding energy of ${ }^{16} \mathrm{O}$ is equal 
to $7.54 \mathrm{Mev} /$ nucleon, which is thought as a reasonable value, given the uncertainties related to the three-nucleon interaction.

Figure 11 shows the theoretical result for the charge form factor in ${ }^{16} \mathrm{O}$. In the one-body Born-approximation picture, the charge form factor is given as

$$
F_{L}(q)=\left\langle\tilde{0}\left|\sum_{k} f_{k}\left(q^{2}\right) e^{i \vec{q} \cdot \vec{r}_{k}^{\prime}}\right| \tilde{0}\right\rangle,
$$

with $f_{k}\left(q^{2}\right)$ the nucleon form factor, which takes into account the finite size of the nucleon $k$ 13. We also take into account the model-independent part of the "Helsinki meson-exchange model" 14, namely the contributions from the $\pi$ - and $\rho$-exchange "seagull" diagrams, with the pion- and $\rho$-meson propagators replaced by the Fourier transforms of the isospin-dependent spin-spin and tensor components of the $v 18 N N$ interaction. This substitution is required in order for the exchange current operator to satisfy the continuity equation together with the interaction model. The contributions of the $\pi$ - and $\rho$-exchange charge density give a measurable correction only for $q>2 \mathrm{fm}^{-1}$.

In order to generate the form factor depicted in Fig. 1, we have first used the procedures of [11], keeping the contributions that can be written in terms of the one- and two-body densities. Then we take the Fourier transform in order to produce the theoretical charge density. Using this theoretical charge density, we generate the charge form factor in a Distorted Wave Born Approximation picture 15], in order to take into account the distortions due to the interaction of the electron with the Coulomb field. This last step results in smoothing out the sharp diffraction minima usually seen in the calculated charge form factor [4,11]. The agreement with the experiment is reasonably good over the whole range of $q$ spanned by the available experimental data [16.

A second electron scattering observable that we would like to compare with is the longitudinal structure function $S_{L}(q)$, sometimes called the Coulomb sum rule, which is sensitive to the short-range correlations induced by the repulsive core of the $N N$ interaction 17, 18]. The Coulomb sum rule, $S_{L}(q)$, represents the total integrated strength of the longitudinal response function measured in inclusive electron scattering. In the nonrelativistic limit [18], we have

$$
S_{L}(q) \equiv 1+\rho_{L L}(q)-\frac{1}{Z}|\langle\tilde{0}|\rho(q)| \tilde{0}\rangle|^{2},
$$

where $\rho(q)$ is the nuclear charge operator

$$
\rho(q)=\frac{1}{2} \sum_{i}^{A} e^{i \vec{q} \cdot \vec{r}_{i}}\left(1+\tau_{z, i}\right)
$$

and $\rho_{L L}(q)$ is the longitudinal-longitudinal distribution function

$$
\rho_{L L}(q)=\int d \vec{r}_{1} \int d \vec{r}_{2} j_{0}\left(q\left|\vec{r}_{1}-\vec{r}_{2}\right|\right) \rho^{(p, p)}\left(\vec{r}_{1}, \vec{r}_{2}\right)
$$

Here $\rho^{(p, p)}\left(\vec{r}_{1}, \vec{r}_{2}\right)$ is the proton-proton two-body density

$$
\begin{aligned}
& \rho^{(p, p)}\left(\vec{r}_{1}, \vec{r}_{2}\right) \\
& =\frac{1}{4} \sum_{i, j}\left\langle\tilde{0}\left|\delta\left(\vec{r}_{1}-\vec{r}_{i}\right) \delta\left(\vec{r}_{2}-\vec{r}_{j}\right)\left(1+\tau_{z, i}\right)\left(1+\tau_{z, j}\right)\right| \tilde{0}\right\rangle
\end{aligned}
$$

normalized as

$$
\int d \vec{r}_{1} \int d \vec{r}_{2} \rho^{(p, p)}\left(\vec{r}_{1}, \vec{r}_{2}\right)=Z-1 .
$$

In light nuclei reasonable agreement between theory and experiment is obtained for the Coulomb sum rule [19]. In heavier nuclei, however, the experimental situation is a lot more controversial, since both a certain lack of strength has been reported and because of the inherent difficulty of separating the longitudinal and transverse contributions in the cross section due to the distortion effects of the electron waves in the nuclear Coulomb field. Figure 2 shows the calculated Coulomb sum in ${ }^{16} \mathrm{O}$. Since no experimental data are available for ${ }^{16} \mathrm{O}$, we compare the results of the present calculation with the ${ }^{12} \mathrm{C}$ experimental data from with an estimate 21] for contributions from large $\omega$. The large error bars on the experimental data are largely due to systematic uncertainties associated with tail contribution [22]. Preliminary theoretical results for ${ }^{12} \mathrm{C}$ are also shown and appear to follow closely the results for theoretical curve for ${ }^{16} \mathrm{O}$.

This calculation represents the most detailed calculation available today, using the coupled cluster expansion, for a nuclear system with $A>8$. This also represents a contribution to the on-going effort of carrying out microscopic calculations that directly produce nuclear shell structure from realistic nuclear interactions. Similar calculations for other closed-shell nuclei in the $p$ - and $s d$ shell are currently under way.

This work was supported in part by the U.S. Department of Energy (DE-FG02-87ER-40371). The work of B.M. was also supported in part by the U.S. Department of Energy under contract number DE-FG0587ER40361 (Joint Institute for Heavy Ion Research), and DE-AC05-96OR22464 with Lockheed Martin Energy Research Corp. (Oak Ridge National Laboratory). The calculations were carried out on a dual-processor $500 \mathrm{MHz}$ Pentium II PC in the Nuclear Physics Group at the University of New Hampshire. Some of the preliminary calculations have also run on a $180 \mathrm{MHz}$ R10000 Silicon Graphics Workstation in the Computational and Theoretical Physics Section at ORNL. The authors gratefully acknowledge useful conversations with John Dawson and David Dean. 
[1] B. S. Pudliner, V. R. Pandharipande, J. Carlson, S. C. Pieper, and R. B. Wiringa, Phys. Rev. C 56, 1720 (1997), nucl-th/9705009; B. S. Pudliner, V. R. Pandharipande, J. Carlson, and R. B. Wiringa, Phys. Rev. Lett. 74, 4396 (1995).

[2] C. R. Chen, G. L. Payne, J. L. Friar, and B. F. Gibson, Phys. Rev. C 31, 2266 (1985); H. Witala, W. Glöckle, and H. Kamada, Phys. Rev. C 43, 1619 (1991).

[3] A. Kievsky, M. Viviani, and S. Rosati, Nucl. Phys. A 551, 241 (1993); A. Kievsky, M. Viviani, and S. Rosati, Nucl. Phys. A 577, 511 (1994).

[4] S. C. Pieper, R. B. Wiringa, and V. R. Pandharipande, Phys. Rev. C 46, 1747 (1992).

[5] H. Kümmel, K. H. Lührmann, and J. G. Zabolitzky, Phys. Reports 36, 1 (1978).

[6] R. B. Wiringa, V. G. Stoks, and R. Schiavilla, Phys. Rev. C 51, 38 (1995).

[7] J. Carlson, V.R. Pandharipande, and R. B. Wiringa, Nucl. Phys. A 401, 59 (1983).

[8] J.H. Heisenberg and B. Mihaila, Phys. Rev. C 59, 1440 (1999), nucl-th/9802029.

[9] J. H. Heisenberg and B. Mihaila, nucl-th/9802031 (1998).

[10] B. Mihaila and J. H. Heisenberg, in preparation.

[11] B. Mihaila and J. H. Heisenberg, nucl-th/9802032 (1998).

[12] P. Navratil and B. R. Barrett, Phys. Rev. C 57, 3119 (1998); D. J. Dean et al., Phys. Rev. C 59, 2474 (1999).

[13] F. Iachello, A. D. Jackson, and A. Lande, Phys. Lett. 43B, 191 (1973).

[14] R. Schiavilla, V. R. Pandharipande, and O. Riska, Phys. Rev. C 41, 309 (1990).

[15] D. R. Yennie, D. G. Ravenhall, and R. N. Wilson, Phy. Rev. 95, 500 (1954).

[16] I. Sick and J.S. McCarthy, Nucl. Phys. A 150, 631 (1970).

[17] K. M. McVoy and L. Van Hove, Phys. Rev. 125, 1034 (1962).

[18] J. Carlson and R. Schiavilla, Rev. Mod. Phys. 70, 743 (1998).

[19] R. Schiavilla, V. R. Pandharipande, and A. Fabrocini, Phys. Rev. C 40, 1484 (1989);

[20] P. Barreau et al., Nucl. Phys. A402, 515 (1983).

[21] R. Schiavilla, A. Fabrocini, and V. R. Pandharipande, Nucl. Phys. A473, 290 (1987).

[22] R. Schiavilla, R. B. Wiringa, and J. Carlson, Phys. Rev. Lett. 70, 3856 (1993).

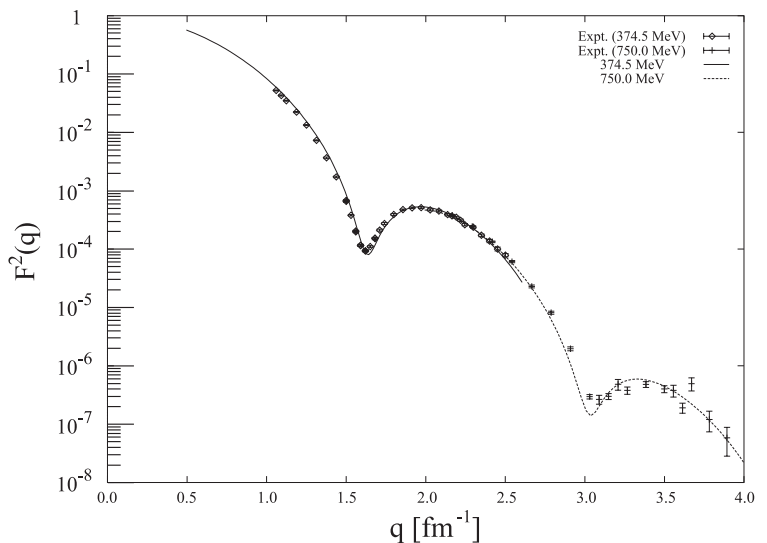

FIG. 1. Charge form factor for O-16, obtained in the DWBA picture $\left(v_{18}+\mathrm{UIX}+\mathrm{ME}\right)$

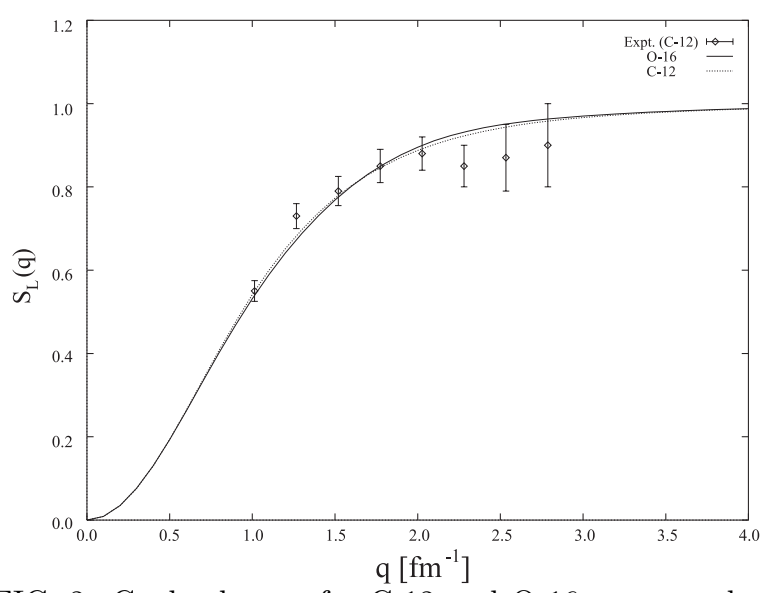

FIG. 2. Coulomb sum for C-12 and O-16, compared with "experimental" C-12 data which include theoretically determined high-energy tail corrections 